\title{
The Changes of Expression Levels in Mir-181a and Mir-30d, and a Significant Correlation between Clinical Patient Data
}

\author{
Mojtaba Mohammadnejad Pahmadani ${ }^{1}$, Fatemeh Jabari ${ }^{1}$, Shima Hojabri Mahani ${ }^{1}$, Reza Mahmanzar ${ }^{2}$ \& Faryad \\ Sharifian ${ }^{1}$ \\ ${ }^{1}$ MSc molecular genetics, Islamic Azad University, Pishva, Varamin, Iran \\ ${ }^{2} \mathrm{MSc}$ molecular genetics, Islamic Azad University, Science and research branch, Tehran, Iran \\ Correspondence: Mojtaba Mohammadnejad pahmadani, MSc molecular genetics, Islamic Azad University, \\ Pishva, Varamin, Iran. E-mail: mojtaba.mohammadnezhad71@gmail.com
}

Received: January 5, 2019 Accepted: January 23, 2019 Online Published: February 23, 2019

doi:10.5539/cco.v8n1p16 URL: https://doi.org/10.5539/cco.v8n1p16

\begin{abstract}
Purpose: Colorectal cancer is known as the most common gastrointestinal cancers. As the age increases, the risk for this cancer also increases, so the only way to improve and hope for life in these patients is early diagnosis of the disease. So far, numerous molecular studies have been carried out on microRNAs in colorectal cancer. In addition, since some of them can be identified as cancer biomarkers. Therefore, in this study we have investigated the expression level of Mir-30d and Mir-181a as cancer biomarkers.
\end{abstract}

Method: The changes of Mir-30d and Mir-181a expression levels in 60 colorectal tumor tissues and 60 adjacent tumor tissues, after RNA extraction and cDNA synthesis were surveyed using the Real Time-PCR method.

Results: The results have reported a considerable reduction in the expression level of Mir-30d in tumor tissues, as well as a significant increase in the expression level of Mir-181a tumor expression in tumor tissues $(\mathrm{P}<0.05)$. In addition, the correlation between Mir-30d and Mir181a showed that there was a significant difference between the level of expression of mir-30d with age and TNM stage of CRC $(\mathrm{P}<0.05)$, whilst these correlations were not observed for Mir-181a ( $\mathrm{P}>0.05)$.

Keywords: colorectal cancer, biomarkers, microRNAs, Mir-30d, Mir-181a, Real Time-PCR

\section{Introduction}

Third cancer worldwide is related to colorectal cancer (CRC). This type of cancer in patients is often diagnosed in advanced stages, so that treatment for these patients in early cases is Surgery (De Rosa et al., 2015). Molecularly, colorectal cancer is a very heterogeneous disease. Loss of genomic stability and increasing multiple mutations facilitates the development of CRCs. Chromosomal instability, aberrant DNA methylation, instability of microsatellite and defect in DNA repair are mechanisms that all involved in CRC (Boland \& Goel, 2010; Goel et al., 2007; Pino \& Chung, 2010; Sideris \& Papagrigoriadis, 2014). About 70\% of sporadic colorectal cancers are due to somatic mutations. Familial Colorectal Cancer is a group of diseases that do not follow Mendelian inheritance. Familial talent for the development of this cancer is about 10-30\%, while its inherited cause is about 5-7\% (Burt, 2000). The highest incidence of colorectal cancer is in the Alaska Natives and blacks communities and the lowest incidence in the Asia/Pacific Islanders. Also, the incidence of this disease in men is 30-40\% higher than in women (Siegel et al., 2017). In 2018, colorectal cancer accounted for about $6.1 \%$ of the world's total cancer mortality, and after lung cancer and prostate cancer, was the most common cancer in men (Bray et al., 2018). In 2017, 95520 cases of colon cancer and 39910 cases of rectal cancer were diagnosed in the United States. So that of 95,520 cases, 47,700 cases of colon cancer related to men and 47,820 cases related to women. Also, of 39910 cases of rectal cancer, 23,720 were men and 16190 were women (https://www.cancer.org). Symptoms of colorectal cancer include changes in bowel habits such as diarrhea or constipation, intestinal bleeding, dark stools due to bleeding and weight loss (Astin, Griffin, Neal, Rose, \& Hamilton, 2011). In recent decades, several studies have been conducted to confirm the existence of diagnostic biomarkers in various cancers. So that many of these studies have pointed to microRNAs for early detection of cancer (X. Chen et al., 2008; Kosaka, Iguchi, \& Ochiya, 2010). Also, multiple studies have been carried out on various microRNAs as an early diagnostic biomarker for colorectal cancer (Aslam, Taylor, Pringle, \& Jameson, 2009). Due to the 
importance of microRNAs in CRC, the expression levels of Mir-30d and Mir-181a were surveyed in this study, while these microRNAs have been investigated in cancers such as breast cancer and gastric cancer (Iliopoulos, Jaeger, Hirsch, Bulyk, \& Struhl, 2010; J. Jiang et al., 2011; Su et al., 2013). The Mir-30 family regulates various processes in normal and cancer tissues such as development (Bridge et al., 2012; Le Guillou et al., 2012), metastasis (Gaziel-Sovran et al., 2011; L. Jiang et al., 2012), apoptosis (J. Li et al., 2010), proliferation (Tanic et al., 2012) and differentiation (Karbiener et al., 2011). Mir-30d has been strengthened in over $30 \%$ of the different types of human epithelial tumors (N. Li et al., 2011; Lu et al., 2009). Mir-30d is one of the targets of AKT signaling. AKT inhibition with a chemical inhibitor or with a siRNA (RNA interference) leads to an increase in the expression level of Mir 30. Also, the analysis indicate that Mir-30d acts as a pro-apoptotic agent and the loss of expression of this microRNA reduces apoptosis (Wu et al., 2013). In addition, Mir-30d and Mir-181a along with GRP78, are regulated in the prostate, colon and bladder tumors (Su et al., 2013). Mir-181a is a member of the Mir family of 181. Studies have shown that Mir-181a by targeting BCL2 plays an important role in the development of MDR (multidrug resistance) in human cancer cells (Zhu, Shan, Wang, Shu, \& Liu, 2010), It also acts as a tumor suppressor in human glioma (Shi et al., 2017). In a study, expression criteria mir-181a indicate the progression of the chronic lymphocytic leukemia (Visone et al., 2011).

\section{Material and Methods}

\subsection{Human Specimens}

Sixty Human colorectal cancer specimens and matched adjacent non-tumor tissues were obtained from patients at Masih Hospital, Tehran, Iran, with informed consent from each patient. $10 \%$ of patient's age was under the 50 years old, $45 \%$ between 50 and 60 years old, $26.7 \%$ between 60 and 70 years and lastly $18.3 \%$ over 70 years. Also, TNM-stage of cancer for tissues was $11.7 \%$ in stage I, 35\% in stage II, $40 \%$ in stage III, and $13.3 \%$ in stage IV. These patients didn't receive any treatment such as chemotherapy or radiation therapy, yet and Clinicopathologic information's of patients are reported in Table 1.

Table 1. Clinicopathologic characteristics of sixty colorectal cancer patients

\begin{tabular}{lc}
\hline Variable & Value (n= 60) \\
\hline Gender & $39(65 \%)$ \\
Male & $21(35 \%)$ \\
Female & \\
Age (years) & $6(10 \%)$ \\
Under 50 (43.7) & $27(45 \%)$ \\
Between 50-60 (57.1) & $16(26.7 \%)$ \\
Between 60-70 (63.9 \pm 0.08$)$ & $11(18.3 \%)$ \\
Over 70 (79.6 \pm 0.06$)$ & \\
Cell type & $60(100 \%)$ \\
Adenocarcinoma & \\
Stage & $7(11.7 \%)$ \\
I & $21(35 \%)$ \\
II & $24(40 \%)$ \\
III & $8(13.3 \%)$ \\
IV &
\end{tabular}

\subsection{RNA Extraction and cDNA Preparation}

For isolation of total RNAs, we using RiboEx (GeneAll, Korea) and RNAs was extracted from all tissues according to the manufacturer's specifications and then, the concentration of extracted RNAs was measured by spectrophotometry and the absorbance 260/280 ratio was qualified between 1.8 to 2.0. The synthesis of cDNA with miRNA-specific stem-loop was performed using the Prime Script RT reagent kit (Takara, Japan) according to the manufacturer's specifications, and at the end, the obtained cDNAs were stored in $-70^{\circ} \mathrm{C}$ until use. The miRNA-specific stem-loop's sequences used for synthesis of cDNA are presented in Table 2.

Table 2. Sequences of miRNA-specific stem-loops used for synthesis of cDNA

\begin{tabular}{lll}
\hline $\begin{array}{l}\text { Target } \\
\text { Name }\end{array}$ & Sequences $\left(\mathbf{5}^{\prime} \rightarrow \mathbf{3}^{\prime}\right)$ & Length \\
\hline Mir-181a-5p & GTCGTATCCAGTGCAGGGTCCGAGGTATTCGCACTGGATACGACACTCAC & $\mathbf{5 0}$ \\
\hline Mir-30d-3p & GTCGTATCCAGTGCAGGGTCCGAGGTATTCGCACTGGATACGACACTCAC & $\mathbf{5 0}$ \\
\hline RNU44 & GTCGTATCCAGTGCAGGGTCCGACCGGTATTCGCACTGGATACGACAGTCAG & $\mathbf{5 2}$ \\
\hline
\end{tabular}




\subsection{Real-time Quantitative PCR}

Real-time PCR was performed using a StepOnePlus ${ }^{\mathrm{TM}}$ Real-Time PCR Systems (ABI Applied Bio-systems, Thermo Fisher Scientific, USA) in a 15- $\mu$ l reaction containing 7.5- $\mu$ l of RealQ Plus 2x Master Mix Green High ROX $^{\mathrm{TM}}$ (Ampliqon, Denmark), $1-\mu \mathrm{l}$ of cDNA, 5.5- $\mu \mathrm{l}$ of $\mathrm{H} 2 \mathrm{O}$ and $1-\mu \mathrm{l}$ of mixed forward and reverse primers (3 Pmol/ $\mu$ l concentration). Real-time PCR amplifications were done as follows: for two selected miRNAs, PCR amplification was set to an initial $95^{\circ} \mathrm{C}$ for $15 \mathrm{~min}$ and then for both miRNAs, a total of 40 cycles, $95^{\circ} \mathrm{C}$ for 15 seconds and $60^{\circ} \mathrm{C}$ for $1 \mathrm{~min}$. All samples were analyzed in duplicate and RNU44 was used as an internal control. The primers used for real-time PCR are reported in Table 3.

Table 3. Primer sequences for selected miRNAs and RNU44

\begin{tabular}{lll}
\hline Target genes & & Sequences $(\mathbf{5} \rightarrow \mathbf{3})$ \\
\hline Mir-181a-5p & Forward & CACGCCAACATTCAACGCTG \\
\cline { 2 - 3 } & Reverse & CCAGTGCAGGGTCCGAGGTA \\
\hline \multirow{2}{*}{ Mir-30d-3p } & Forward & CACGCAAGTAAACATCCCCGAC \\
\cline { 2 - 3 } & Reverse & CCAGTGCAGGGTCCGAGGTA \\
\hline \multirow{2}{*}{ RNU44 } & Forward & CCTGGATGATGATAGCAAATG \\
\cline { 2 - 3 } & Reverse & TCGTATCCAGTGCAGGG \\
\hline
\end{tabular}

\subsection{Statistical Analysis}

Gene expression was estimated using the comparative threshold cycle $\left(2^{-}{ }^{-}{ }^{\mathrm{CT}}\right)$ method and T-test was used to compared expression in tumor and controls group. All tests, was performed using the GraphPad Prism software v7.03 (GraphPad Software Inc., USA) and $P$ value $<0.05$ was considered statistically significant.

\section{Results}

\subsection{The Outcomes of Mir-30d Expression in Colorectal Cancer Samples Using q-PCR Method}

The results obtain of Mir-30d expression in 60 normal and tumor tissues demonstrated that there is a significant difference between the expression level of this microRNA in tumor tissues compared with normal tissues $(\mathrm{P}=0.0014)$. According to Figure 1, $39(65 \%)$ samples of 60 tumor samples showed a decrease in Mir-30d expression level. Whilst, 21 (35\%) tumor samples reported an increase in Mir-30d expression level. lastly, we have reported a significant reduction in the expression level of Mir-30d in colorectal tumor tissues through statistical surveys.

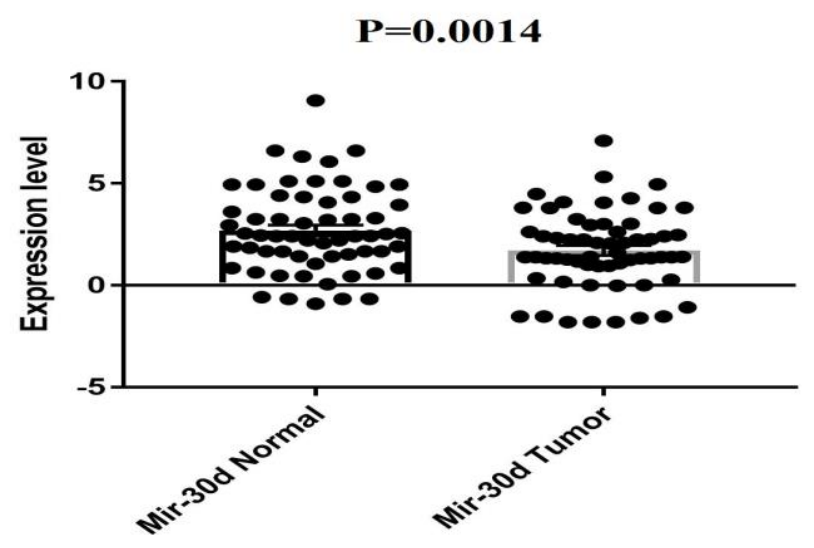

Figure 1. The graph is related to comparison of Mir-30d expression level between tumor and normal tissues

\subsection{The Outcomes of Mir-181a Expression in Colorectal Cancer Samples Using q-PCR Method}

According to the results of Mir-181a (Figure 2), out of max 60 tumor tissues, the increase of Mir-181a expression level and the reduce of Mir-181a expression level were observed in 41 (68.3\%) tumor tissues and 19 (31.7\%) tumor tissues, respectively. Therefore, the expression level of Mir-181a increased dramatically in CRC, which indicates a considerably difference in the expression level of this miRNA in colorectal tumor tissues compared with normal tissues $(\mathrm{P}=0.01)$. 


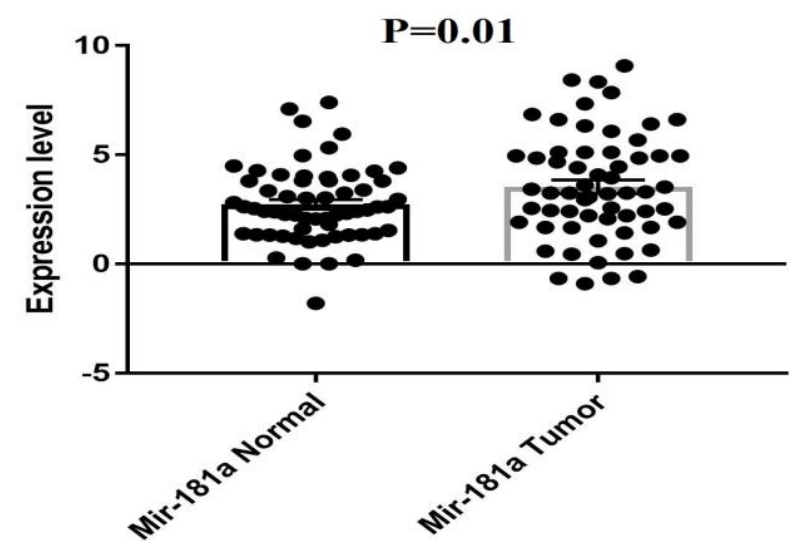

Figure 2. The graph is related to comparison of Mir-181a expression level between tumor and normal tissues

\subsection{Correlation between Mir-30d, Mir-181a Expression and Clinic-pathologic Parameters}

Using statistical analyzes, the correlation was examined between the expression level of Mir-30d, Mir-181a against gender, age, and TNM-stage of CRC. According to the results of Mir-30d (Table 4), there is a significant difference between the reduction of the Mir-30d expression level and age and stage of cancer. It means that samples which have been at the advanced stage of cancer and had higher ages have illustrated the lower expression than early-stage and lower ages samples $(\mathrm{P}<0.05)$. With regard to Table 4 , a significant correlation was not found between the increase of Mir-181a expression and clinic-pathologic parameters $(\mathrm{P}>0.05)$.

Table 4. Correlation between Mir-30d, Mir-181a expression and clinicopathologic parameters in the patients with $\mathrm{CRC}$

\begin{tabular}{|c|c|c|c|c|c|c|c|}
\hline \multirow[t]{2}{*}{ Variable } & \multirow[t]{2}{*}{ N0. of cases } & \multirow{2}{*}{\multicolumn{2}{|c|}{$\begin{array}{l}\text { Mir30d expression } \\
\text { High (21) Low (39) }\end{array}$}} & \multirow[t]{2}{*}{ P value } & \multirow{2}{*}{\multicolumn{2}{|c|}{ High(41) Low(19) }} & \multirow[t]{2}{*}{ P value } \\
\hline & & & & & & & \\
\hline Gender & & & & 0.312 & & & 0.261 \\
\hline Male & 39 & 16 & 23 & & 29 & 10 & \\
\hline Female & 21 & 5 & 16 & & 12 & 9 & \\
\hline Age (years) & & & & 0.037 & & & 0.082 \\
\hline Under 60 & 33 & 12 & 21 & & 20 & 13 & \\
\hline Over 60 & 27 & 9 & 18 & & 21 & 6 & \\
\hline Stage & & & & 0.043 & & & 0.074 \\
\hline I- II & 28 & 13 & 15 & & 18 & 10 & \\
\hline III- IV & 32 & 8 & 24 & & 23 & 9 & \\
\hline
\end{tabular}

\section{Discussion}

CRC is one of the most widespread malignancies with high morbidity and mortality, all over the world. Many people die each year due to the late diagnosis of the disease, and patients continue to suffer from a low survival rate. Finding out the factors involved in this type of cancer and detecting potential diagnostic markers is the best way to increase the survival rate of these patients. In recent years, one of the areas that could attract a lot of attention is deregulation of miRNAs during cancer initiation and progression, which provides trustworthy knowledge for the distinct comprehension of molecular mechanisms underlying CRC. Due to the role miRNA played in the signaling pathway, the expression abnormalities of miRNA can also affect a wide range of target genes and eventually cellular activities, such as metastasis, migration, proliferation, and invasion. Thus, major research of the miRNAs involved in the advancement of CRC may contribute to making better diagnostics and more effectual therapeutic strategies for CRC patients.

Earlier research on Mir-30d determines multiple results in various cancers. Kobayashi et al, by studying on the expression level of Mir-30d in normal and cancer prostate tissues demonstrated that Mir-30d was overexpressed in prostate cancer tissues, and they also nominated it as a novel prognostic marker for prostate cancer (Kobayashi et al., 2012). In another study, Chen et al. showed that Mir-30d expression was reduced in non-small cell lung cancer tissues compared to normal lung tissues (D. Chen et al., 2015). In this study, we analyzed the expression 
level of Mir-30d in 60 colorectal cancer patients and we demonstrated that Mir-30d was downregulated in CRC tissues compared to control group. According to the roles that Mir-30d plays in many processes, such cell proliferation, induction of cell cycle at the G0/G1 phase, invasion and migration (Wu et al., 2013). It's clear that dysregulation of Mir-30d can disturb the normal cellular functions and causes the onset and progression of the CRC. Analysis of the clinicopathological parameters showed that the miR-30d expression level was associated with age and advanced TNM stage. These data suggest the Mir-30d has an important and crucial role in the initiation and progression of CRC and it can be used as a diagnostic marker and potential molecular therapeutic target.

Another miRNA that was investigated in this study was Mir-181a, and it has already been studied in various cancers. Shi et al. showed that Mir-181a was downregulated in non-small cell lung cancer and cell lines and it's significantly associated with histological grade and TNM stage (Shi et al., 2017). In contrast, miR-181a expression was dramatically and selectively upregulated in metastatic breast tumors (Taylor, Sossey-Alaoui, Thompson, Danielpour, \& Schiemann, 2013). In this paper, we demonstrated that the expression level of Mir-181a was significantly increased in CRC tissues compared to normal colorectal tissues. According to past functional studies, Mir-181a plays a significant role in the EMT process and it's clear that overexpression of Mir-181a causing reduce expression of the epithelial maker and increase expression of the mesenchymal maker. It can also be used as a diagnostic and therapeutic marker if it has other features.

\section{Conclusions}

In this paper, we analyzed the expression level of Mir-30d and Mir-181a in 60 colorectal cancer patients and we demonstrated that Mir-30d was downregulated in CRC tissues compared to control group which is associated with age and advanced TNM stage. In contrast, the expression level of Mir-181a was significantly increased in CRC tissues compared to normal colorectal tissues. Mir-30d and Mir-181a can also be used as a diagnostic and therapeutic marker if it has other features.

\section{References}

Aslam, M., Taylor, K., Pringle, J., \& Jameson, J. (2009). MicroRNAs are novel biomarkers of colorectal cancer. British Journal of Surgery: Incorporating European Journal of Surgery and Swiss Surgery, 96(7), 702-710. https://doi.org/10.1002/bjs.6628

Astin, M., Griffin, T., Neal, R. D., Rose, P., \& Hamilton, W. (2011). The diagnostic value of symptoms for colorectal cancer in primary care: a systematic review. Br J Gen Pract, 61(586), e231-e243. https://doi.org/10.3399/bjgp11X572427

Boland, C. R., \& Goel, A. (2010). Microsatellite instability in colorectal cancer. Gastroenterology, 138(6), 2073-2087. e2073. https://doi.org/10.1053/j.gastro.2009.12.064

Bray, F., Ferlay, J., Soerjomataram, I., Siegel, R. L., Torre, L. A., \& Jemal, A. (2018). Global cancer statistics 2018: GLOBOCAN estimates of incidence and mortality worldwide for 36 cancers in 185 countries. CA: $a$ cancer journal for clinicians, 68(6), 394-424. https://doi.org/10.3322/caac.21492

Bridge, G., Monteiro, R., Henderson, S., Emuss, V., Lagos, D., Georgopoulou, D., ... Boshoff, C. (2012). The microRNA-30 family targets DLL4 to modulate endothelial cell behavior during angiogenesis. Blood, blood-2012-2004-423004. https://doi.org/10.1182/blood-2012-04-423004

Burt, R. W. (2000). Colon cancer screening. Gastroenterology, 119(3), 837-853. https://doi.org/10.1053/gast.2000.16508

Chen, D., Guo, W., Qiu, Z., Wang, Q., Li, Y., Liang, L., ... He, X. (2015). MicroRNA-30d-5p inhibits tumour cell proliferation and motility by directly targeting CCNE2 in non-small cell lung cancer. Cancer letters, 362(2), 208-217. https://doi.org/10.1016/j.canlet.2015.03.041

Chen, X., Ba, Y., Ma, L., Cai, X., Yin, Y., Wang, K., ... Guo, X. (2008). Characterization of microRNAs in serum: a novel class of biomarkers for diagnosis of cancer and other diseases. Cell research, 18(10), 997.

De Rosa, M., Pace, U., Rega, D., Costabile, V., Duraturo, F., Izzo, P., \& Delrio, P. (2015). Genetics, diagnosis and management of colorectal cancer. Oncology reports, 34(3), 1087-1096. https://doi.org/10.3892/or.2015.4108

Gaziel-Sovran, A., Segura, M. F., Di Micco, R., Collins, M. K., Hanniford, D., de Miera, E. V.-S., ... Kerbel, R. S. (2011). miR-30b/30d regulation of GalNAc transferases enhances invasion and immunosuppression during metastasis. Cancer cell, 20(1), 104-118. https://doi.org/10.1016/j.ccr.2011.05.027 
Goel, A., Nagasaka, T., Arnold, C. N., Inoue, T., Hamilton, C., Niedzwiecki, D., ... Bertagnolli, M. M. (2007). The $\mathrm{CpG}$ island methylator phenotype and chromosomal instability are inversely correlated in sporadic colorectal cancer. Gastroenterology, 132(1), 127-138. https://doi.org/10.1053/j.gastro.2006.09.018

Iliopoulos, D., Jaeger, S. A., Hirsch, H. A., Bulyk, M. L., \& Struhl, K. (2010). STAT3 activation of miR-21 and miR-181b-1 via PTEN and CYLD are part of the epigenetic switch linking inflammation to cancer. Molecular cell, 39(4), 493-506. https://doi.org/10.1016/j.molcel.2010.07.023

Jiang, J., Zheng, X., Xu, X., Zhou, Q., Yan, H., Zhang, X., ... Ju, J. (2011). Prognostic significance of miR-181b and miR-21 in gastric cancer patients treated with S-1/Oxaliplatin or Doxifluridine/Oxaliplatin. PloS one, 6(8), e23271.

Jiang, L., Lin, C., Song, L., Wu, J., Chen, B., Ying, Z., ... Li, J. (2012). MicroRNA-30e* promotes human glioma cell invasiveness in an orthotopic xenotransplantation model by disrupting the NF- $\mathrm{KB} / \mathrm{I} \kappa \mathrm{B} \alpha$ negative feedback loop. The Journal of clinical investigation, 122(1), 33-47.

Karbiener, M., Neuhold, C., Opriessnig, P., Prokesch, A., Bogner-Strauss, J. G., \& Scheideler, M. (2011). MicroRNA-30c promotes human adipocyte differentiation and co-represses PAI-1 and ALK2. RNA biology, 8(5), 850-860. https://doi.org/10.4161/rna.8.5.16153

Kobayashi, N., Uemura, H., Nagahama, K., Okudela, K., Furuya, M., Ino, Y., ... Aoki, I. (2012). Identification of miR-30d as a novel prognostic maker of prostate cancer. Oncotarget, 3(11), 1455.

Kosaka, N., Iguchi, H., \& Ochiya, T. (2010). Circulating microRNA in body fluid: a new potential biomarker for cancer diagnosis and prognosis. Cancer science, 101(10), 2087-2092. https://doi.org/10.1111/j.1349-7006.2010.01650.x

Le Guillou, S., Sdassi, N., Laubier, J., Passet, B., Vilotte, M., Castille, J., ... Jaffrézic, F. (2012). Overexpression of miR-30b in the developing mouse mammary gland causes a lactation defect and delays involution. PloS one, 7(9), e45727. https://doi.org/10.1371/journal.pone.0045727

Li, J., Donath, S., Li, Y., Qin, D., Prabhakar, B. S., \& Li, P. (2010). miR-30 regulates mitochondrial fission through targeting p53 and the dynamin-related protein-1 pathway. PLoS genetics, 6(1), e1000795. https://doi.org/10.1371/journal.pgen.1000795

Li, N., Kaur, S., Greshock, J., Lassus, H., Zhong, X., Wang, Y., ... Liang, S. (2011). A combined array-based comparative genomic hybridization $(\mathrm{aCGH})$ and functional library screening approach identifies mir-30d as an oncomir in cancer. Cancer research, canres. 2484.2011. https://doi.org/10.1158/0008-5472.CAN-11-2484

Lu, Y., Ryan, S. L., Elliott, D. J., Bignell, G. R., Futreal, P. A., Ellison, D. W., ... Clifford, S. C. (2009). Amplification and overexpression of Hsa-miR-30b, Hsa-miR-30d and KHDRBS3 at 8q24. 22-q24. 23 in medulloblastoma. PloS one, 4(7), e6159. https://doi.org/10.1371/journal.pone.0006159

Pino, M. S., \& Chung, D. C. (2010). The chromosomal instability pathway in colon cancer. Gastroenterology, 138(6), 2059-2072. https://doi.org/10.1053/j.gastro.2009.12.065

Shi, Q., Zhou, Z., Ye, N., Chen, Q., Zheng, X., \& Fang, M. (2017). MiR-181a inhibits non-small cell lung cancer cell proliferation by targeting CDK1. Cancer Biomarkers (Preprint), 1-8. https://doi.org/10.3233/CBM-170350

Sideris, M., \& Papagrigoriadis, S. (2014). Molecular biomarkers and classification models in the evaluation of the prognosis of colorectal cancer. Anticancer research, 34(5), 2061-2068.

Siegel, R. L., Miller, K. D., Fedewa, S. A., Ahnen, D. J., Meester, R. G., Barzi, A., \& Jemal, A. (2017). Colorectal cancer statistics, 2017. CA: a cancer journal for clinicians, 67(3), 177-193. https://doi.org/10.3322/caac.21387

Su, S., Chang, Y., Andreu-Vieyra, C., Fang, J., Yang, Z., Han, B., ... Liang, G. (2013). miR-30d, miR-181a and miR-199a-5p cooperatively suppress the endoplasmic reticulum chaperone and signaling regulator GRP78 in cancer. Oncogene, 32(39), 4694.

Tanic, M., Yanowsky, K., Rodriguez-Antona, C., Andrés, R., Márquez-Rodas, I., Osorio, A., ... Martinez-Delgado, B. (2012). Deregulated miRNAs in hereditary breast cancer revealed a role for miR-30c in regulating KRAS oncogene. PloS one, 7(6), e38847. https://doi.org/10.1371/journal.pone.0038847

Taylor, M. A., Sossey-Alaoui, K., Thompson, C. L., Danielpour, D., \& Schiemann, W. P. (2013). TGF- $\beta$ upregulates miR-181a expression to promote breast cancer metastasis. The Journal of clinical investigation, 
123(1), 150-163.

Visone, R., Veronese, A., Rassenti, L. Z., Balatti, V., Pearl, D. K., Acunzo, M., ... Croce, C. M. (2011). miR-181b is a biomarker of disease progression in chronic lymphocytic leukemia. Blood, blood-2011-2001-333484. https://doi.org/10.1182/blood-2011-01-333484

Wu, C., Jin, B., Chen, L., Zhuo, D., Zhang, Z., Gong, K., \& Mao, Z. (2013). MiR-30d induces apoptosis and is regulated by the Akt/FOXO pathway in renal cell carcinoma. Cellular signalling, 25(5), 1212-1221. https://doi.org/10.1016/j.cellsig.2013.01.028

Zhu, W., Shan, X., Wang, T., Shu, Y., \& Liu, P. (2010). miR-181b modulates multidrug resistance by targeting BCL2 in human cancer cell lines. International Journal of Cancer, 127(11), 2520-2529.

https://doi.org/10.1002/ijc.25260

\section{Copyrights}

Copyright for this article is retained by the author(s), with first publication rights granted to the journal.

This is an open-access article distributed under the terms and conditions of the Creative Commons Attribution license (http://creativecommons.org/licenses/by/4.0/). 